\title{
Effect of trimethoprim-sulphamethoxazole on the risk of malaria in HIV-infected Ugandan children living in an area of widespread antifolate resistance
}

\author{
Anne F Gasasira*1,3, Moses R Kamya1', Edwin O Ochong2, Neil Vora22, Jane Achan1', Edwin Charlebois22, Theodore Ruel2, \\ Fredrick Kateera', Denise N Meya', Diane Havlir², Philip J Rosenthal² and Grant Dorsey²
}

\begin{abstract}
Background: Daily trimethoprim-sulfamethoxazole (TS) protects against malaria, but efficacy may be diminished as anti-folate resistance increases. This study assessed the incidence of falciparum malaria and the prevalence of resistance-conferring Plasmodium falciparum mutations in HIV-infected children receiving daily TS and HIV-uninfected children not taking TS.

Materials and methods: Subjects were $292 \mathrm{HIV}$-infected and 517 uninfected children from two cohort studies in Kampala, Uganda observed from August 2006 to December 2008. Daily TS was given to HIV-infected, but not HIVuninfected children and all participants were provided an insecticide-treated bed net. Standardized protocols were used to measure the incidence of malaria and identify markers of antifolate resistance.

Results: Sixty-five episodes of falciparum malaria occurred in HIV-infected and 491 episodes in uninfected children during the observation period. TS was associated with a protective efficacy of $80 \%$ ( $0.10 \mathrm{vs}$. 0.45 episodes per person year, $\mathrm{p}<0.001)$, and efficacy did not vary over three consecutive 9.5 month periods $(81 \%, 74 \%, 80 \%$ respectively, $\mathrm{p}=$ 0.506). The prevalences of $d h f r 511,108 \mathrm{~N}$, and 59R and dhps $437 \mathrm{G}$ and 540E mutations were each over $90 \%$ among parasites infecting both HIV-infected and uninfected children. Prevalence of the dhfr $164 \mathrm{~L}$ mutation, which is associated with high-level resistance, was significantly higher in parasites from HIV-infected compared to uninfected children ( $8 \%$ vs. $1 \%, p=0.001)$. Sequencing of the $d h f r$ and $d h p s$ genes identified only one additional polymorphism, dhps 581G, in 2 of 30 samples from HIV-infected and 0 of 54 samples from uninfected children.
\end{abstract}

Conclusion: Despite high prevalence of known anti-folate resistance-mediating mutations, TS prophylaxis was highly effective against malaria, but was associated with presence of $d h f r 164 \mathrm{~L}$ mutation.

\section{Background}

Daily prophylaxis with the anti-folate combination trimethoprim-sulphamethoxazole (TS) has been shown to reduce morbidity and improve survival in HIVinfected populations [1,2]. In addition to the prevention of opportunistic and other common bacterial infections associated with HIV infection, TS reduces the risk of malaria in HIV-infected adults and children living in subSaharan Africa [1,3-5]. The World Health Organization, UNAIDS and UNICEF recommend TS prophylaxis for all HIV-infected patients with symptomatic disease (WHO

\footnotetext{
* Correspondence: agasasira@gmail.com

1 School of Medicine, Makerere University Kampala, Uganda Full list of author information is available at the end of the article
}

Stage 2, 3, and 4), Stage 1 HIV-infected adults with CD4 lymphocyte counts less than 350 cells/ $\mu$ l, Stage 1 HIVinfected children with CD4 lymphocyte percentage less than $25 \%$, and all infants born to HIV-infected mothers (HIV-exposed) [6].

Despite the evidence for significant benefits associated with TS use in HIV-infected patients, implementation of TS prophylaxis in sub-Saharan Africa remains low. A recent UNICEF report estimated that only $4 \%$ of the 4 million children in need of TS prophylaxis in sub-Saharan Africa are receiving this intervention [7]. There are concerns that in areas where anti-folate resistance is extensive, TS may not be effective in preventing malaria and bacterial infections, while in areas where resistance is not 
yet widespread, expanded TS use may lead to the rapid selection and spread of Plasmodium falciparum parasites resistant to anti-folates. This in turn may diminish the preventive efficacy of TS and sulphadoxinepyrimethamine (SP), which acts against the same two enzyme targets as TS. Cross-resistance between TS and SP is of particular concern as, although SP is being replaced by more effective agents for the treatment of malaria, it remains the only recommended drug for intermittent preventive therapy (IPT), a validated measure to prevent malaria in pregnant women [8].

To characterize the risks and benefits of daily TS, this study compared the incidence of malaria and the prevalence of anti-folate resistance-conferring mutations in $P$. falciparum infections in HIV-uninfected children not taking TS and HIV-infected children taking daily TS in an area of moderate malaria transmission. Further, the effect of time on the protective efficacy of TS and the prevalence of anti-folate resistance-conferring mutations was evaluated.

\section{Methods Study site}

The study was carried out in Kampala, Uganda. Kampala is an urban setting in south-central Uganda where malaria is mesoendemic, occurring perennially with peaks following two rainy seasons, and an entomological inoculation rate (EIR) estimated to be less than 10 infective bites per person year in 2005 (unpublished data).

\section{Study subjects}

Subjects were children participating in two parallel cohort studies, which have been described previously $[4,9]$ and are summarized here briefly. The first study enrolled 599 healthy children presumed to be HIV-uninfected (432 tested HIV negative, 167 HIV test results unavailable; all subjects were healthy) into a communitybased cohort from a geographically defined census population using probability sampling from November 2004 through April 2005. Eligibility criteria included: 1) age 110 years, 2) agreement to come to the study clinic for any febrile episode or other illness, 3) agreement to remain in Kampala for the duration of the study, 4) agreement to avoid medications administered outside the study protocol, 5) lack of history of any known serious chronic disease requiring frequent medical attention, including HIV/AIDS, 6) weight $>10 \mathrm{~kg}$, and 7) provision of informed consent from parent or guardian. None of the HIV-uninfected cohort study participants were prescribed TS prophylaxis. All study participants were given an insecticide treated bed net (ITN) between May-June 2006. The second study enrolled 300 HIV-infected children from a pediatric HIV clinic in Kampala from October 2005 through August 2006 using convenience sampling. Eligibility criteria were the same as for the HIV-uninfected cohort, except for: 1) living within a 20 $\mathrm{km}$ radius of the study clinic, 2) no restriction based on a history of serious chronic disease, and 3) weight $>5 \mathrm{~kg}$. All HIV-infected cohort participants were prescribed daily TS and provided with an ITN at enrollment.

\section{Study participant follow-up}

Similar protocols were used for follow-up of both cohorts. Subjects were followed for all of their medical problems in dedicated study clinics open seven days a week, with after hours care available at Mulago Hospital. Children who presented with new medical problems underwent standardized medical evaluation. A set of algorithms was developed to guide therapy for common illnesses. Medications with anti-malarial activity were avoided for the treatment of non-malarial illnesses when possible. HIV-infected subjects meeting standard WHO eligibility criteria were provided antiretroviral therapy according to local guidelines[10]. The preferred first line therapy for those initiated to ART was Efavirenz (EFV), lamivudine (3TC) and zidovudine (AZT). Subjects who presented to the clinics with a documented fever (tympanic temperature $>38.0^{\circ} \mathrm{C}$ ) or history of fever in the previous 24 hours had blood obtained by fingerprick for a thick blood smear. If the thick blood smear was positive, the patient was diagnosed with malaria regardless of the parasite density. HIV-uninfected participants diagnosed with uncomplicated malaria were assigned randomly to antimalarial treatment with sulphadoxine-pyrimethamine plus amodiaquine $(\mathrm{SP}+\mathrm{AQ})$, amodiaquine plus artesunate $(\mathrm{AQ}+\mathrm{AS})$, or artemether-lumefantrine (AL). HIVinfected children were treated with AQ + AS between August 2006 and June 2007. Use of AQ + AS in this study was stopped after a high risk of neutropaenia secondary to malaria treatment was observed [11]; it was replaced with AL from July 2007 to the end of the study. All episodes of complicated malaria and treatment failures occurring within 14 days of initial therapy were treated with quinine.

TS adherence in HIV-infected participants was measured during routine monthly visits using caregivers' selfreport of administering drugs during the previous 3 days.

Study participants were withdrawn from follow-up if any of the following occurred: 1) movement out of study area for $>60$ consecutive days, 2) inability to be located for $>60$ consecutive days, 3) withdrawal of informed consent, or 4) inability to adhere to study schedule and procedures.

\section{Laboratory methods}

Thick smears were stained with $2 \%$ Giemsa for 30 minutes. Parasite density was estimated by counting the number of asexual parasites per 200 white blood cells and 
calculating parasites per $\mu \mathrm{L}$, assuming a white blood cell count of 8,000 cells per $\mu \mathrm{L}$. A smear was judged to be negative if no parasites were seen after review of 100 high-powered fields. Final microscopy results were based on a rigorous quality control system which included rereading all blood smears by a second microscopist and resolution of any discrepancies between the first and second readings by a third microscopist. Parasite species on the day malaria was diagnosed was determined using nested PCR of $18 \mathrm{~S}$ small sub-unit ribosomal DNA as previously described [12].

For repeat episodes of malaria in the same study participant, molecular genotyping was used to distinguish new from recrudescent infections. DNA was isolated from blood samples collected on filter paper on the day of malaria diagnosis, and samples were genotyped in a stepwise fashion by assessing polymorphisms in $m s p-2, m s p$ 1 , and four microsatellites as previously described [13]. If, for any of the six loci, an allele was not shared between consecutive episodes of malaria, the episode was classified as a new infection. If at least one allele was shared between consecutive episodes of malaria at all six loci, the episode was classified as a recrudescence.

Samples were tested on the day malaria was diagnosed for the following polymorphisms associated with antifolate resistance: dihydrofolate reductase (dhfr) N51I, C59R, S108N, and I164L; and dihydropteroate synthetase (dhps) A437G and K540E. Polymorphisms were identified using a nested polymerase chain reaction followed by sequence-specific restriction enzyme digestion. Primers, amplification conditions, and restriction endonucleases for these assays were as previously described [14,15]. Digestion products were visualized by agarose gel electrophoresis and results were classified according to the migration patterns of the fragments. Laboratory investigators were blinded to clinical data at the time of molecular analysis.

For a subset of samples, the complete $d h f r$ and $d h p s$ genes were amplified by nested PCR and sequenced. For $d h f r$, the gene was amplified with primers 3A (5'-CCCAAATAGCTAGTTCAGGGGAAC and 4B (5'-TCATCTTCTTCTTCATCATCATCATC) followed by the nested primers 3'A (5'-CTCCTTTTTATGATGGAACAAGTC TGCG) and 3B (5'-TGACATGTATCTTTGTCATCAT TC) to yield a $785 \mathrm{bp}$ fragment.

For dhps, primers were 6A (5'-GGATCAGAAGATGAATAATCATGT) and 3B (5'-CACTAAACCATTAGAGTACTTGAC), followed by 7A (5'-CCATTCCTC ATGTGTATACAACAC) and 5B (5'-GTTTAATCACATGTTTGCACTTTC-3'), yielding a 1326 bp fragment.

Amplicons were purified using Exosap (USB) and sequenced bi-directionally using nested primers at the Genomics Core Facility, UCSF. Sequence data were aligned and manually curated using Lasergene (DNASTAR).

\section{Statistical analysis}

The period of observation began on August 1, 2006 (when enrollment for both cohorts was complete and all study participants had been given an ITN) and continued through December 15, 2008 (when follow-up for the HIV-uninfected cohort ended) or the date of premature study withdrawal.

Data were entered and verified using Access (Microsoft Corporation, Redmond, WA). Analyses were performed using STATA version 10.0 (Stata Corp., College Station, TX). Log-transformed parasite densities were compared using the two-sample t-test and categorical variables (comparisons of mutation prevalence and parasite species) were analysed using Fisher exact or chi-square tests. Malaria incidence was defined as the number of new episodes of malaria (recrudescences were excluded) per period of observation. Negative binomial regression models were used to measure the association between use of TS prophylaxis and malaria incidence (expressed as an incidence rate ratio (IRR)), controlling for age. The protective efficacy of TS was defined as 1 - IRR. Tests for trend were used to assess for changes in the protective efficacy of TS and the prevalence of anti-folate resistanceconferring mutations over the following time periods: August 12006 to May 15 2007; May 162007 to February 292008 and March 12008 to December 15 2008. A pvalue $<0.05$ was considered statistically significant.

\section{Ethical approval}

Written informed consent was obtained from the parents/guardians of children for their participation in the study. Ethical approval for the two cohort studies was obtained from the Uganda National Council of Science and Technology, Makerere University Research and Ethics Committee, and the University of California, San Francisco Committee on Human Research.

\section{Results}

\section{Study participants}

Characteristics of study participants are shown in Table 1. Of the 599 participants enrolled into the HIV-uninfected cohort, 517 were still in active follow-up by August 2006 and included in the study. Likewise, 292 of the 300 HIV-infected children initially enrolled were active and included in the present study. The median level of TS adherence in the HIV-infected population over the study period was $100 \%$, with only 216 of 8257 (3\%) assessments associated with less than $100 \%$ adherence. The mean age at the beginning of the observation period was significantly higher for HIV-uninfected compared to HIVinfected participants (7.4 vs. 5.7 years, $\mathrm{p}<0.0001)$, pri- 
Table 1: Patient characteristics

\begin{tabular}{lcc}
\hline Characteristics & HIV-uninfected* & HIV-infected* \\
\hline Number of subjects & 517 & 292 \\
Total person years of observation & 1095 & 665 \\
Median duration of follow up in yrs (range) & $2.1(0.2-2.4)$ & $2.4(0-2.4)$ \\
Mean age in yrs at the start of follow-up (SD) & $7.4(2.7)$ & N/A \\
Mean CD4 percent at start of follow-up (SD) & N/A & $23 \%(8.6)$ \\
Antiretroviral (ART) use during follow-up & N/A & 275 \\
$\quad$ Total person years on ART & N/A & $76(26 \%)$ \\
$\quad$ Number of participants on ART at start of follow-up & $65(22 \%)$ \\
$\quad$ Number of participants initiated on ART during follow-up & \\
\hline
\end{tabular}

*All HIV-uninfected children were not receiving TS while all HIV-infected children were receiving daily TS

marily because the HIV-infected cohort was recruited about one year later than the uninfected cohort. A total of 452 (87\%) HIV-uninfected children and 277 (94\%) HIVinfected children were followed for the full observation period. The mean CD4 cell percentage in the HIVinfected cohort was $23 \%$ at baseline, $76(26 \%)$ participants received anti-retroviral therapy (ART) throughout the study period, and $65(22 \%)$ were initiated on ART during follow-up.

\section{Characteristics of malaria episodes}

The large majority of treatments for malaria were for new, rather than recrudescent infections, as expected for cohorts in which malaria episodes were treated with highly efficacious combination regimens (Table 2). Ninety-two percent of new malaria episodes were due to P. falciparum in both HIV-uninfected and HIV-infected children. The geometric mean parasite density of malaria infections was lower for HIV-infected (6,462 parasites/ $\mu$ l) compared to HIV-uninfected $(11,270$ parasites/ $\mu \mathrm{l})$ children, however this difference was not statistically significant $(\mathrm{p}=0.397)$.

\section{Malaria incidence}

The protective efficacy of TS for the entire observation period was $80 \%$ (95\% CI $72-85 \%$ ) adjusting for age at the start of the study, and efficacy did not decline over the 3 consecutive 9.5-months periods that constituted the observation period [IRRs: 0.19 (0.12-0.28), 0.26 (0.15$0.43)$ and $0.20(0.10-0.38)$, respectively (Table 3$)]$. The protective efficacy of TS was similar for HIV-infected participants receiving $[76 \%$ (95\% C.I. $63-84 \%)]$ and not receiving ART [83\% (95\% C.I 74-89\%)].

\section{Prevalence of resistance-mediating polymorphisms}

In new $P$. falciparum infections, the prevalences of five $d h f r$ and dhps point mutations known to mediate diminished response to therapy with SP and to be common in

Table 2: Characteristics of malaria episodes

\begin{tabular}{lcc}
\hline Characteristics & $\begin{array}{c}\text { HIV-uninfected* } \\
\text { (n= 517) }\end{array}$ & $\begin{array}{c}\text { HIV-infected } \\
\text { (n= 292) }\end{array}$ \\
\hline Number of treatments for malaria & 511 & 65 \\
New episodes of malaria ${ }^{\dagger}$ & 491 & 65 \\
Species for new episodes & & $60(92 \%)$ \\
$\quad$ P. falciparum & \\
P. malariae & $451(92 \%)$ & $2(3 \%)$ \\
P. ovale & $23(4 \%)$ & $3(5 \%)$ \\
P. vivax & $14(3 \%)$ & 0 \\
Geometric means parasite density per $\mu \mathrm{L}^{\S}$ & $3(1 \%)$ & 6462 \\
\hline
\end{tabular}

* All HIV-uninfected participants were not receiving TS and all HIV-infected participants were receiving daily TS

${ }^{\dagger}$ After exclusion of episodes due to recrudescences identified by genotyping

* Includes $P$. falciparum mono and mixed infections

$\S$ Only include new episodes of falciparum malaria 
Table 3: Comparison of malaria incidence

\begin{tabular}{|c|c|c|c|c|c|c|c|}
\hline \multirow[t]{2}{*}{ Time Period } & \multicolumn{3}{|c|}{ HIV-uninfected } & \multicolumn{3}{|c|}{ HIV-infected } & \multirow[t]{2}{*}{ IRR (95\% C.I) } \\
\hline & Episodes & Person time (yrs) & Incidence & Episodes & Person time & Incidence & \\
\hline Aug $1^{\text {st }} 06-$ Dec $15^{\text {th }} 08$ & 491 & 1095 & 0.45/psnyr & 65 & 665 & 0.10/psnyr & $0.20(0.15-0.28)$ \\
\hline Aug $1^{\text {st }} 06-$ May $15^{\text {th }} 07$ & 267 & 397 & $0.67 / p s n y r$ & 28 & 224 & $0.13 / p s n y r$ & $0.19(0.12-0.28)$ \\
\hline May $16^{\text {th }} 07-$ Feb $29^{\text {th }} 08$ & 132 & 364 & $0.36 / p s n y r$ & 23 & 222 & $0.10 / p s n y r$ & $0.26(0.15-0.43)$ \\
\hline Mar $1^{\text {st }} 08-\operatorname{Dec} 15^{\text {th }} 08$ & 92 & 334 & $0.28 / p s n y r$ & 14 & 220 & $0.06 /$ psnyr & $0.20(0.10-0.38)$ \\
\hline
\end{tabular}

*Incident Rate Ratio controlling for age at beginning each observation period

East Africa [16] (dhfr 51I, 108N, and C59R and dhps 437G and $540 \mathrm{E}$ ) were all over $90 \%$, independent of TS use (Table 4) and time. The simultaneous presence of all 5 of these mutations was also common for HIV-uninfected and infected participants $(86 \%$ vs. $92 \%, \mathrm{p}=0.222)$ and prevalence did not increase significantly over the 3 consecutive 9.5-month periods in HIV-infected $(96 \%, 86 \%$, $93 \%$ respectively, $\mathrm{p}=0.446)$ or uninfected $(82 \%, 89 \%, 88 \%$ respectively, $\mathrm{p}=0.467$ ) participants.

In contrast to the mutations noted above, $d h f r 164 \mathrm{~L}$, which has been associated with high-level SP resistance, was uncommon. Of note, this mutation was significantly more common in HIV-infected compared to HIV-uninfected participants ( $8 \%$ vs. $1 \%, p=0.001)$. As with the other mutations, prevalence of the $d h f r 164 \mathrm{~L}$ mutation did not change with time in parasites from HIV-infected $(12 \%, 10 \%, 0 \% \mathrm{p}=0.417)$ or uninfected participants $(0 \%$, $2 \%, 0 \%, \mathrm{p}=0.375)$.

To assess for novel mutations in the $d h f r$ or $d h p s$ genes as a result of daily TS use, the complete genes were sequenced in a subset of samples, including $d h f r$ from 40 and dhps from $30 \mathrm{HIV}$-infected children receiving TS and dhfr from 73 and dhps from 54 uninfected children not receiving TS. Sequencing confirmed the sequences at known polymorphic alleles in all samples but one, in which $d h f r 108 \mathrm{~N} / 51 \mathrm{I} / 164 \mathrm{~L}$ mutations were seen in the initial analysis and 108N/51I/59R seen with sequencing; presumably the discrepancy was due to characterization of different strains in a mixed infection. Sequencing identified only one additional polymorphism, the previously reported dhps 581G mutation, which was seen in samples from 2 of 30 children receiving daily TS and none of the 54 HIV-uninfected children for whom sequence results were available $(\mathrm{p}=0.125)$.

\section{Discussion}

The presence of simultaneous cohorts including HIVinfected children receiving daily TS and HIV-uninfected children not receiving TS allowed for the assessment of the anti-malarial preventive efficacy of TS. Results from

Table 4: Prevalence of molecular markers of anti-folate resistance among new episodes of $P$. falciparum malaria

\begin{tabular}{|c|c|c|c|}
\hline Characteristics & $\begin{array}{l}\text { HIV-uninfected* } \\
\quad(n=352)\end{array}$ & $\begin{array}{l}\text { HIV-infected* } \\
\quad(n=60)\end{array}$ & P-value \\
\hline \multicolumn{4}{|c|}{ Prevalence of $d h f r$ mutations } \\
\hline 511 & $179(99 \%)^{\dagger}$ & $60(100 \%)$ & 0.563 \\
\hline $59 \mathrm{R}$ & $163(91 \%)^{\dagger}$ & $56(93 \%)$ & 0.510 \\
\hline $108 \mathrm{~N}$ & $180(100 \%)^{\dagger}$ & $60(100 \%)$ & \\
\hline $164 \mathrm{~L}$ & $1(1 \%)^{\dagger}$ & $5(8 \%)$ & 0.001 \\
\hline \multicolumn{4}{|c|}{ Prevalence of $d h p s$ mutations } \\
\hline $437 G$ & $172(96 \%)^{\dagger}$ & $58(97 \%)$ & 0.709 \\
\hline $540 \mathrm{E}$ & $174(97 \%)^{\dagger}$ & 59 (99\%) & 0.506 \\
\hline Quintuple mutation ${ }^{\ddagger}$ & $154(86 \%)^{\dagger}$ & $55(92 \%)$ & 0.222 \\
\hline
\end{tabular}

*All HIV-uninfected participants were not receiving TS and all HIV-infected participants were receiving daily TS

${ }^{+}$Assessed in a random sample of 180 episodes matched by malaria treatment and calendar time

‡dhfr 511/59R/108N + dhps $437 \mathrm{G} / 540 \mathrm{E}$ mutations 
this study show that daily TS was highly efficacious in conferring protection against malaria in HIV-infected children despite a high prevalence of resistance-mediating $P$. falciparum polymorphisms. This study adds to a previous one by this group which reported a $97 \%$ reduction in malaria risk in HIV-infected children associated with the combined use of TS and ITNs [4]. In the present study, without the confounder of ITN use in only the HIV-infected children, the protective efficacy of daily TS was more clearly defined, and it remained high.

TS use has recently been shown to protect against malaria in adults [3] and children [4] in Uganda, but it has not been clear if continued protection will be seen with increasing prevalence of mutations in the target enzymes $d h f r$ and $d h p s$ that limit anti-malarial treatment efficacy of the related anti-folate SP [17-20]. However, in the present study, despite some of the highest prevalences of 5 key $d h f r$ and $d h p s$ mutations reported in Africa, TS offered strong protective efficacy against malaria, and protective efficacy was maintained throughout the 29month observation period.

Drugs that inhibit the folate pathway enzymes $d h f r$ and dhps have important roles in the treatment of many infections. TS is active against many bacteria and opportunistic pathogens, leading to recommendations for its widespread use in HIV-infected and exposed individuals in Africa. SP, which inhibits the same two enzymes as TS, has been a standard anti-malarial therapy in Africa. Use of SP to treat malaria is now discouraged due to increasing resistance, especially in Southern and East Africa, with resistance mediated by step-wise selection and also selective sweeps of 5 key mutations [21]. Additional mutations in $d h f r$ and $d h p s$, notably $d h f r 164 \mathrm{~L}$, mediate higher levels of resistance, but these mutations are uncommon in Africa [22]. Despite decreasing malaria treatment efficacy, SP retains a key role in malaria control, as it is the only drug well-established to protect against malaria when used as IPT in pregnant women [23] or infants [24]. In summary, the anti-folates TS and SP remain critical elements of control efforts for HIV infection and malaria, but resistance-mediating mutations are common in P. falciparum in many areas, and key questions remain inadequately answered. This study considered the following three major concerns regarding daily use of TS in HIV-infected individuals.

First, did daily TS offer protection against malaria despite the presence of anti-folate resistance-mediating mutations? Five key resistance-mediating mutations, which were already known to be common in much of East Africa [25], were remarkably common in parasites isolated from infected patients in this study. In this setting, daily TS nonetheless offered strong preventive efficacy against malaria. Thus, although the resistance-mediating mutations may have mitigated the protective efficacy of
TS to some extent, the intervention nonetheless had a pronounced effect. This result bodes well for Africa, where the prevalence of these 5 key mutations is increasing, but other mutations that mediate higher-level resistance remain uncommon.

Second, did TS use select for parasites with additional anti-folate resistance mediating mutations that might ablate any protective efficacy of anti-folates? Blood samples were screened for the $d h f r$ 164L mutation, which is common in parts of South America and Asia [21], but has not shown high prevalence in Africa. Recently, however, the mutation was seen in $4 \%$ of parasites isolated in Western Kenya [26], in 4.7\% of parasites in Malawi from subjects receiving SP [27], and 4-14\% of symptomatic subjects not receiving anti-folates in two low transmission highland regions of Western Uganda [28]. In this study $d h f r$ 164L was uncommon in HIV-uninfected children who did not receive TS. However, compared to those who did not receive the intervention, prevalence of the $d h f r 164 \mathrm{~L}$ mutation was significantly greater in HIVinfected children who received TS. These results indicate that, in certain settings, daily TS may select for $d h f r 164 \mathrm{~L}$. The clinical significance of selection for this additional mutation is unknown, but biochemical studies have shown profound resistance in parasites harboring $d h f r$ 164L [29]. However, it remains unclear whether the $d h f r$ $164 \mathrm{~L}$ mutation will spread in Africa. Indeed, as is the case with some other resistance-mediating polymorphisms that may engender a fitness cost (e.g. increased copy number of the putative drug transporter gene $p f m d r 1$ ), the high level of anti-malarial immunity of populations in areas of high malaria transmission in Africa may limit selection for $d h f r$ 164L [18]. The complete $d h f r$ and $d h p s$ genes were sequenced in a subset of samples and only one additional polymorphism, the dhps 581G mutation, was identified in 2 samples from children receiving daily TS. This mutation has been associated with high-level in vitro sulphadoxine resistance in P. falciparum [30], has been seen in Southeast Asia and South America [30,31], and had a prevalence of $55 \%$ in a recent study in northern Tanzania [32]. In Kampala, the prevalence of the $581 \mathrm{G}$ mutation was low, and it is unclear if it was selected by daily TS. Importantly, no novel $d h f r$ or $d h p s$ mutations were identified.

Third, did the protective efficacy of TS decline over time? In this study, TS efficacy was maintained over the 29-month observation period. This is not surprising given the stability of the anti-folate-mediating mutations assessed. Five of the 6 mutations evaluated were close to saturated throughout the study period, while the prevalence of the 164L mutation, which is not yet widespread, did not increase in those exposed or unexposed to TS. The evolution of anti-folate resistance likely occurred over several years, limiting the ability of the study to 
detect changes over the relatively short observation period. Therefore, while the present study gives an indication that TS prophylactic efficacy may be sustained in areas where background anti-folate resistance is extensive, more studies will be needed to assess its longer-term efficacy.

This study had some limitations. For ethical reasons, HIV-infection and TS use could not be separated. Therefore, comparisons of TS-unexposed and exposed participants were between HIV-uninfected children not receiving TS and HIV-infected children receiving the intervention. Sixteen percent of presumed HIV-uninfected subjects were not tested for HIV, as they had either been excluded from the cohort prior to testing or declined to be tested. However, the lack of HIV infection among the 432 children who were tested suggests that very few of the cohort, if any, had this infection, and any bias resulting from misclassification of HIV status would be expected to underestimate differences seen between the HIV-infected and HIV-uninfected cohorts. The impact of TS on 6 well characterized polymorphisms in $d h f r$ and dhps was considered in all samples, but due to logistical constraints whole gene sequences were assessed in only a subset of samples chosen based on amplification and sequencing success; sequencing of only a portion of the total might have introduced bias, and so it is possible that other rare polymorphisms were missed. Finally, the study was done in a relatively low transmission setting, and findings may not be generalizable to other settings, particularly those with higher malaria transmission intensity. These limitations, however, do not alter the key conclusions of the study, as noted above.

\section{Conclusions}

Considering impacts on malaria, this study strongly supports the use of TS prophylaxis in HIV-infected children in Africa. Further, given the excellent protective efficacy of TS, consideration might be given for its use in populations at highest risk of adverse consequences of malaria in addition to other transmission reduction measures such as ITNs. The study was not well-equipped to assess the impact of daily TS on the selection of common anti-folate resistance-mediating mutations, as these were already very common at our study site. The findings of this study, together with those from studies assessing SP efficacy for IPTp, suggest that the prophylactic efficacies of TS and SP remain high in even where anti-folate resistance is widespread. However, the selection of an additional mutation, $d h f r 164 \mathrm{~L}$, in children receiving daily TS is concerning, and suggests the need for continued surveillance to monitor the protective efficacy of TS and the prevalence of resistance-mediating polymorphisms.

\section{About the Authors}

PJR is a Doris Duke Charitable Foundation Distinguished Clinical Scientist.
Competing interests

The authors declare that they have no competing interests.

\section{Authors' contributions}

MRK, DH, PJR, GD, EC, and AFG contributed to the design of the study. MRK, JA, DM, FK, NV, and AFG supervised enrollment and follow-up of study participants and directed the clinical studies. NV and EO performed the molecular studies. MRK, DH, PRJ, TR, EC and GD participated in the oversight of study activities. GD and AFG verified and analysed the data. All authors assisted with interpretation of the data and preparation of the manuscript and approved the final report.

\section{Acknowledgements}

We are grateful to the $\mathrm{NIH}$ for funding the cohort studies and to the Forgarty International Center for training support. We thank all the parents/guardians for giving their consent and the study participants for their cooperation. We thank our three study teams including the clinic team: Nestor Mbabazi, Caroline Akello, Tamara Clark, Catherine Maiteki, Bridget Nzarubara, Julia Mwesigwa; the data laboratory quality-control, field and administrative teams.

Funding support: This study received financial support from the National Institute of Health (Al052142 \& Al062677)

\section{Author Details}

1School of Medicine, Makerere University Kampala, Uganda, ${ }^{2}$ Department of Medicine, San Francisco General Hospital, University of California, San Francisco, USA and ${ }^{3}$ Department of Epidemiology, University of California, Berkeley, USA

Received: 2 February 2010 Accepted: 23 June 2010

Published: 23 June 2010

\section{References}

1. Anglaret $X$, Chene $G$, Attia A, Toure S, Lafont S, Combe P, Manlan K, N'DriYoman T, Salamon R: Early chemoprophylaxis with trimethoprimsulphamethoxazole for HIV-1-infected adults in Abidjan, Côte d'Ivoire: a randomised trial. Cotrimo-CI Study Group. Lancet 1999, 353:1463-1468

2. Wiktor SZ, Sassan-Morokro M, Grant AD, Abouya L, Karon JM, Maurice C, Djomand G, Ackah A, Domoua K, Kadio A Yapi A, Combe P, Tossou O, Roels TH, Lackritz EM, Coulibaly D, De Cock KM, Coulibaly IM, Greenberg AE: Efficacy of trimethoprim-sulphamethoxazole prophylaxis to decrease morbidity and mortality in HIV-1-infected patients with tuberculosis in Abidjan, Côte d'Ivoire: a randomised controlled trial. Lancet 1999, 353:1469-1475.

3. Mermin J, Ekwaru JP, Liechty CA, Were W, Downing R, Ransom R, Weidle P, Lule J, Coutinho A, Solberg P: Effect of co-trimoxazole prophylaxis, antiretroviral therapy, and insecticide-treated bednets on the frequency of malaria in HIV-1-infected adults in Uganda: a prospective cohort study. Lancet 2006, 367:1256-1261.

4. Kamya MR, Gasasira AF, Achan J, Mebrahtu T, Ruel T, Kekitiinwa A, Charlebois ED, Rosenthal PJ, Havlir D, Dorsey G: Effects of trimethoprimsulfamethoxazole and insecticide-treated bednets on malaria among HIV-infected Ugandan children. AIDS 2007, 21:2059-2066.

5. Thera MA, Sehdev PS, Coulibaly D, Traore K, Garba MN, Cissoko Y, Kone A Guindo A, Dicko A, Beavogui A, Djimde AA, Lyke KE, Diallo DA, Doumbo OK, Plowe CV: Impact of trimethoprim-sulfamethoxazole prophylaxis on falciparum malaria infection and disease. J Infect Dis 2005, 192:1823-1829.

6. WHO: Guidelines on co-trimoxazole prophylaxis for HIV-related infections among children, adolescents and adults. Recommendations for a public health approach 2006 [http://www.who.int/hiv/pub/guidelines/ ctxguidelines.pdf]

7. WHO UU: Children and AIDS: a stocktaking report 20072007 [http:// data.unaids.org/pub/report//2007/20060116 stocktaking report.pdf].

8. Garner P, Gulmezoglu AM: Drugs for preventing malaria in pregnant women. Cochrane Database Syst Rev 2006:CD000169.

9. Davis JC, Clark TD, Kemble SK, Talemwa N, Njama-Meya D, Staedke SG, Dorsey G: Longitudinal study of urban malaria in a cohort of Ugandan children: description of study site, census and recruitment. Malar J 2006, 5:18.

10. Ministry of Health U: Uganda National Antiretroviral Treatment and Care Guidelines for Adults and Children 2003

11. Gasasira AF, Kamya MR, Achan J, Mebrahtu T, Kalyango JN, Ruel T, Charlebois E, Staedke SG, Kekitiinwa A, Rosenthal PJ, Havlir D, Dorsey G: 
High risk of neutropenia in HIV-infected children following treatment with artesunate plus amodiaquine for uncomplicated malaria in Uganda. Clin Infect Dis 2008, 46:985-991.

12. Snounou G, Viriyakosol S, Zhu XP, Jarra W, Pinheiro L, do Rosario VE, Thaithong S, Brown KN: High sensitivity of detection of human malaria parasites by the use of nested polymerase chain reaction. Mol Biochem Parasitol 1993, 61:315-320.

13. Greenhouse B, Myrick A, Dokomajilar C, Woo JM, Carlson EJ, Rosenthal PJ, Dorsey G: Validation of microsatellite markers for use in genotyping polyclonal Plasmodium falciparum infections. Am J Trop Med Hyg 2006, 75:836-842.

14. Plowe CV, Djimde A, Bouare M, Doumbo O, Wellems TE: Pyrimethamine and proguanil resistance-conferring mutations in Plasmodium falciparum dihydrofolate reductase: polymerase chain reaction methods for surveillance in Africa. Am J Trop Med Hyg 1995, 52:565-568.

15. Duraisingh MT, Curtis J, Warhurst DC: Plasmodium falciparum: detection of polymorphisms in the dihydrofolate reductase and dihydropteroate synthetase genes by PCR and restriction digestion. Exp Parasitol 1998, 89:1-8.

16. Gregson A, Plowe CV: Mechanisms of resistance of malaria parasites to antifolates. Pharmacol Rev 2005, 57:117-145.

17. Ndounga M, Tahar R, Basco LK, Casimiro PN, Malonga DA, Ntoumi F: Therapeutic efficacy of sulfadoxine-pyrimethamine and the prevalence of molecular markers of resistance in under 5-year olds in Brazzaville, Congo. Trop Med Int Health 2007, 12:1164-1171.

18. Mugittu K, Ndejembi M, Malisa A, Lemnge M, Premji Z, Mwita A, Nkya W, Kataraihya J, Abdulla S, Beck HP, Mshinda H: Therapeutic efficacy of sulfadoxine-pyrimethamine and prevalence of resistance markers in Tanzania prior to revision of malaria treatment policy Plasmodium falciparumdihydrofolate reductase and dihydropteroate synthase mutations in monitoring in vivo resistance. Am J Trop Med Hyg 2004, 71:696-702.

19. Swarthout TD, van den Broek IV, Kayembe G, Montgomery J, Pota H Roper C: Artesunate + amodiaquine and artesunate + sulphadoxinepyrimethamine for treatment of uncomplicated malaria in Democratic Republic of Congo: a clinical trial with determination of sulphadoxine and pyrimethamine-resistant haplotypes. Trop Med Int Health 2006, 11:1503-1511

20. Alifrangis M, Enosse S, Khalil IF, Tarimo DS, Lemnge MM, Thompson R, Bygbjerg IC, Ronn AM: Prediction of Plasmodium falciparum resistance to sulfadoxine/pyrimethamine in vivo by mutations in the dihydrofolate reductase and dihydropteroate synthetase genes: a comparative study between sites of differing endemicity. Am J Trop Med Hyg 2003, 69:601-606.

21. Sibley CH, Hyde JE, Sims PF, Plowe CV, Kublin JG, Mberu EK, Cowman AF, Winstanley PA, Watkins WM, Nzila AM: Pyrimethamine-sulfadoxine resistance in Plasmodium falciparum: what next? Trends Parasitol 2001 17:582-588

22. Nzila A, Ochong E, Nduati E, Gilbert K, Winstanley P, Ward S, Marsh K: Why has the dihydrofolate reductase 164 mutation not consistently been found in Africa yet? Trans R Soc Trop Med Hyg 2005, 99:341-346.

23. ter Kuile FO, van Eijk AM, Filler SJ: Effect of sulfadoxine-pyrimethamine resistance on the efficacy of intermittent preventive therapy for malaria control during pregnancy: a systematic review. JAMA 2007, 297:2603-2616

24. Kobbe R, Adjei S, Kreuzberg C, Kreuels B, Thompson B, Thompson PA, Marks F, Busch W, Tosun M, Schreiber N, Opoku E, Adjei O, Meyer CG, May $\mathrm{J}$ : Malaria incidence and efficacy of intermittent preventive treatment in infants (IPTi). Malar J 2007, 6:163.

25. Francis D, Nsobya SL, Talisuna A, Yeka A, Kamya MR, Machekano R, Dokomajilar C, Rosenthal PJ, Dorsey G: Geographic differences in antimalarial drug efficacy in Uganda are explained by differences in endemicity and not by known molecular markers of drug resistance. $J$ Infect Dis 2006, 193:978-986.

26. McCollum AM, Poe AC, Hamel M, Huber C, Zhou Z, Shi YP, Ouma P, Vulule J, Bloland P, Slutsker L, Barnwell JW, Udhayakumar V, Escalante AA: Antifolate resistance in Plasmodium falciparum: multiple origins and identification of novel dhfr alleles. J Infect Dis 2006, 194:189-197.

27. Alker AP, Mwapasa V, Purfield A, Rogerson SJ, Molyneux ME, Kamwendo DD, Tadesse E, Chaluluka E, Meshnick SR: Mutations associated with sulfadoxine-pyrimethamine and chlorproguanil resistance in
Plasmodium falciparum isolates from Blantyre, Malawi. Antimicrob Agents Chemother 2005, 49:3919-3921.

28. Lynch C, Pearce R, Pota H, Cox J, Abeku TA, Rwakimari J, Naidoo I, Tibenderana J, Roper C: Emergence of a dhfr Mutation Conferring HighLevel Drug Resistance in Plasmodium falciparum Populations from Southwest Uganda. J Infect Dis 2008, 197:1598-1604.

29. Watkins WM, Mberu EK, Winstanley PA, Plowe CV: The efficacy of antifolate antimalarial combinations in Africa: a predictive model based on pharmacodynamic and pharmacokinetic analyses. Parasitol Today 1997, 13:459-464.

30. Brooks DR, Wang P, Read M, Watkins WM, Sims PF, Hyde JE: Sequence variation of the hydroxymethyldihydropterin pyrophosphokinase: dihydropteroate synthase gene in lines of the human malaria parasite, Plasmodium falciparum, with differing resistance to sulfadoxine. Eur $J$ Biochem 1994, 224:397-405.

31. McCollum AM, Mueller K, Villegas L, Udhayakumar V, Escalante AA: Common origin and fixation of Plasmodium falciparum dhfr and dhps mutations associated with sulfadoxine-pyrimethamine resistance in a low-transmission area in South America. Antimicrob Agents Chemother 2007, 51:2085-2091.

32. Gesase S, Gosling RD, Hashim R, Ord R, Naidoo I, Madebe R, Mosha JF, Joho A, Mandia V, Mrema H, Mapunda E, Savael Z, Lemnge M, Mosha FW, Greenwood B, Roper C, Chandramohan D: High resistance of Plasmodium falciparum to sulphadoxine/pyrimethamine in northern Tanzania and the emergence of dhps resistance mutation at Codon 581. PLoS One 2009, 4:e4569.

doi: 10.1186/1475-2875-9-177

Cite this article as: Gasasira et al., Effect of trimethoprim-sulphamethoxazole on the risk of malaria in HIV-infected Ugandan children living in an area of widespread antifolate resistance Malaria Journal 2010, 9:177

\section{Submit your next manuscript to BioMed Centra and take full advantage of:}

- Convenient online submission

- Thorough peer review

- No space constraints or color figure charges

- Immediate publication on acceptance

- Inclusion in PubMed, CAS, Scopus and Google Scholar

- Research which is freely available for redistribution 